\title{
TRAGALDABAS:
}

\section{a high performance detector for the regular study of cosmic ray properties}

\author{
Juan A. Garzón for the TRAGALDABAS Collaboration* ${ }^{\dagger}$ \\ LabCAF, F. Física. Univ. of Santiago de Compostela \\ E-mail: juanantonio.garzon@usc.es
}

\begin{abstract}
In order to deepen the understanding of the properties of cosmic rays arriving to the Earth, a new generation high granularity and high time resolution cosmic ray tracking detector, TRAGALDABAS, based on timing RPC cells, has been recently installed at the Faculty of Physics of the Univ. of Santiago de Compostela, in Spain. At the same time a broad multidisciplinary collaboration, with researchers of several European countries, has been organized for both the maintenance and the improvement of the facility and for the analysis of the data. The detector is taking data with three active planes since March. 2015 at an approximate rate of $80 \mathrm{~Hz}$. In this article, some of the performances and technical aspects of the detector areas are commented and some preliminary results related with several of the accessible research are discussed.
\end{abstract}

The 34th International Cosmic Ray Conference,

30 July- 6 August, 2015

The Hague, The Netherlands

${ }^{*}$ Speaker.

${ }^{\dagger}$ See full list of members of the TRAGALDABAS Colloboration at the end of the article 


\section{Introduction}

Cosmic rays of a wide range of energies are arriving regularly to the Earth atmosphere coming from the Sun, our galaxy and far galaxies. Before and after arriving to the Earth atmosphere, cosmic rays are affected by different effects, as the interplanetary magnetic field induced by the Sun, the solar wind, the Earth's magnetic field or the atmosphere. All these effects, on the one hand, make more difficult to measure the properties of the primary cosmic rays but, on the other hand, may provide valuable information about them. Fig. 1 summarizes most of the knowledge we have about the primary cosmic rays as a function of their energy: where do they come from, how are they affected by the interplanetary magnetic field, the magnetic field of the Earth and the solar modulation as well as how they interact in the high atmosphere producing bundles of up to billions of secondary particles.

Recently, a detailed analysis of the space-time micro-structure of cosmic ray showers done with the detectors of the RPC ToF wall of the HADES experiment at GSI showed several interesting features yet not well understood [1]. A striking by product of the studies was that, apparently, a high time resolution $(\sim 0.1 \mathrm{~ns})$ and high granularity detector, even having a surface of barely 1 $\mathrm{m}^{2}$, may provide a good estimate of the arrival direction of the front of the shower and perhaps, using both the time width and the density information, make a rough estimation of the energy of the primary cosmic ray. If this is the case, such a detector could be used for the regular scan of the arrival of cosmic rays at a broad range of energies, allowing to analyse a big variety of phenomena.

With all these ideas in mind, a new generation tracking detector based on the RPC ToF wall of the HADES experiment at GSI, TRAGALDABAS [2], has been installed at the Univ. of Santiago de Compostela for the regular study of cosmic rays (Fig. 2). The detector may be sensitive, among others, to the following fields of research:

- Study of the micro-structure of the high energy cosmic ray air showers and search for new observables

- Regular survey of the solar activity and Space Weather phenomena

- Analysis of the Earth's magnetic field

- Analysis of the influence of the temperature of the stratosphere in the rate of cosmic rays on the ground and search for possible correlations between cosmic rays and the climate

In section 2 we describe the main technical features of the detector. In section 3 some of the aspects related with the decoding and reconstruction software and with the slow control tasks already implemented in the detector will be provided. In section 4 we present some preliminary results obtained with the detector.

\section{The detector}

TRAGALDABAS (TRAsGo for the AnaLysis of the nuclear matter Decay, the Atmosphere, the earth B-Field And the Solar activity) is based on the RPC (Resistive Plate Chamber) technology. It is inspired on the RPC ToF wall of the HADES experiment at the GSI, from which it uses some of the digitizing and data acquisition components. 
In its present layout, the detector covers a surface of $1.2 \times 1.5 \mathrm{~m}^{2}$ and has a height of $1.8 \mathrm{~m}$. It is composed by four planes of RPC cells, with two $1 \mathrm{~mm}$-gap each placed inside a gas tight metacrilate box, filled with flowing freon R134a. The readout is done by $12011 \times 11 \mathrm{~cm}^{2}$ external pads. The four planes are placed at the heights: $180 \mathrm{~cm}, 120 \mathrm{~cm}, 90 \mathrm{~cm}$ and $0 \mathrm{~cm}$. At this moment, only the first, the second and the fourth planes are now fully instrumented. The acquisition is triggered by coincidences between planes at $0 \mathrm{~cm}$ and at $120 \mathrm{~cm}$. These features provide an angular resolution better than $2^{\circ}$ at all the zenith angles and a relative uncertainty in the speed of the particles of $\sim 5 \%$. This unusual performance allows even to measure protons arriving to the detector. A new trigger board is being developed that would allow, in the future, to trigger the acquisition by coincidences between any two plane combination.

All these features do allow the detector to reconstruct the time-space micro-structure of the cosmic rays front. Another uncommon performance of the detector is that, unlike other cosmic ray detectors, it doesn't make use of any absorber which makes it sensitive to any of the charged secondary cosmic rays and high energy gammas. We expect that the muon and the electromagnetic components of a shower can be statistically dissagregated using software algorithms. Fig 3 an example of the tracking capability of the detector.

The detector is installed on the first floor of a two-floor building, that will reject part of the EM component of the showers, in the geographical coordinates ( $42^{\circ} 52^{\prime} 34 "$ ", W $8^{\circ} 33^{\prime} 37^{\prime \prime}$ ) and at $\sim 260 \mathrm{~m}$ over the sea level (Fig. 4). This location makes very difficult to make absolute measurements of the lowest energy cosmic ray showers. In order to overcome this problem, it is foreseen to install a replica of the detector outdoors at $\sim 100 \mathrm{~m}$ distance. The detector is taking regularly data at a rate of about 7 million registered events per day $(\sim 80 \mathrm{~Hz})$.

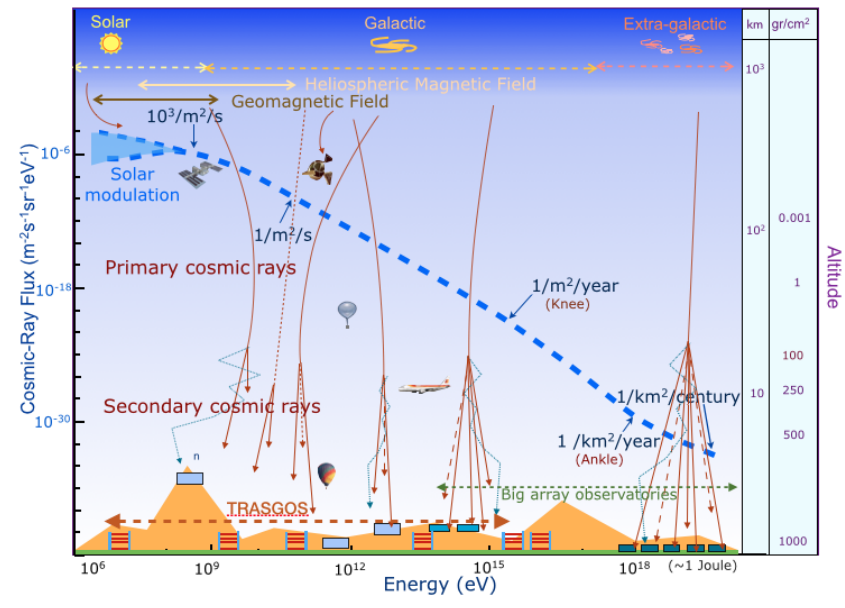

Figure 1: Energy spectrum of primary cosmic rays with their corresponding estimated sources and the main detection techniques. Trasgo could be useful up to energies around the knee

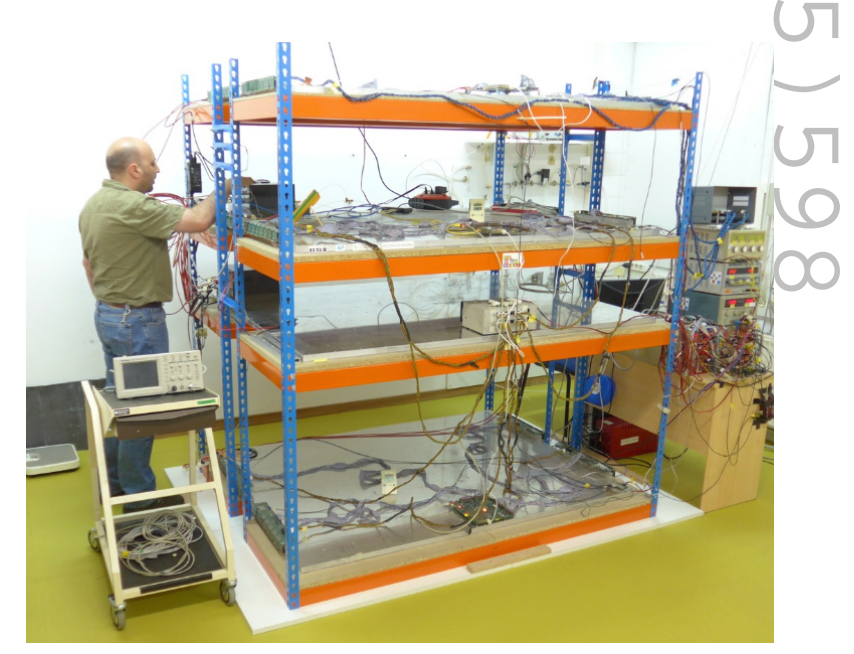

Figure 2: Present layout of the Trasgo TRAGALDABAS at the Univ. of Santiago de Compostela; at this moment, only three planes are fully instrumented.

TRAGALDABAS uses the same Front End Electronics (FEE) design that is used at the HADES spectrometer at GSI, Darmstadt [6] since 2009. RPC signals are first amplified and integrated and then digitized in LVDS format in 4-channels daughter boards (DB). Every 8 DBs are housed in a motherboard (MB), that provides them the low voltage and the output for both the digital read-out 
and the trigger signals. The four MBs collecting all the signals of a RPC plane are read-out by a TDC based read-out board (TRB) [9]. The detector-"acquisition electronics" chain provides a joint time resolution of $\sim 280 \mathrm{ps}$ and an efficiency close to $100 \%$. Data is collected in the acquisition computer and is regularly sent to the supercomputing center $\mathrm{CESGA}^{1}$, for its definitive storage. In the near future the main counting rate values will be accessible in a web site for their public use.

As a summary, in its present layout, the main performances of the detector are:

- No. of instrumented planes: 3

- Granularity: 120 pads / plane; pad size: $130 \mathrm{~cm}^{2}$.

- Angular resolution: $1.5^{\circ}-2^{\circ}$

- Hit time resolution: $\sim 340$ ps.

- Track time resolution: $\sim 180 \mathrm{ps.}$

- Velocity resolution for speed of light particles: $<6 \%$

- Hit efficiency: $\sim 1$

- Data acquisition rate: $\sim 80 \mathrm{~Hz}$.

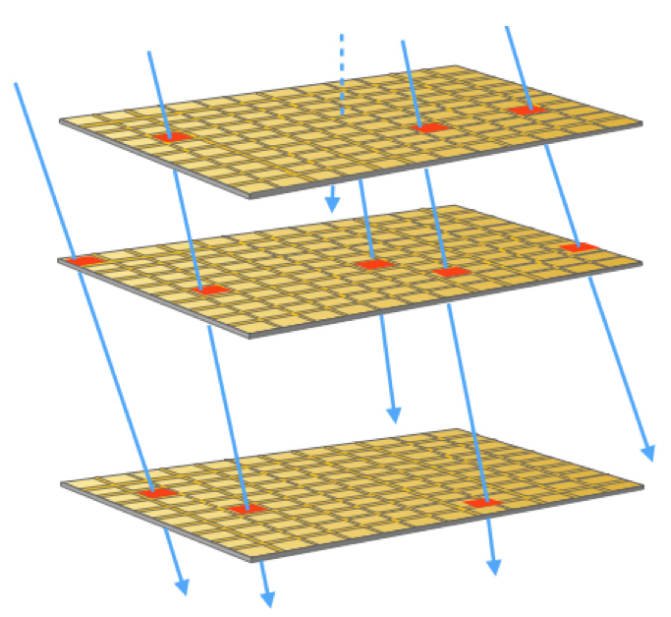

Figure 3: Example of the detection, tracking and identfication capabilities of TRAGALDABAS. Once an event has been triggered all the hits are stored for their analysis.



Figure 4: Location of TRAGALDABAS at the Faculty of Physics building of the Univ. of Santiago de Compostela

\section{Monitoring, Calibration and Simulation Software}

\subsection{Monitoring}

The subsystems of the slow control system at TRAGALDABAS experiment can be classified in three different subgroups, the high voltage subsystem (HV) that feeds the RPC planes, the internal RPC plane sensors which measure the temperature, pressure and humidity inside the RPC planes and the environmental sensors that will measure the temperature both inside and outside the laboratory and the ambient pressure. All the data generated by these subsystems is collected by a Nagios service [8], a well known open source solution initially developed to monitor resources

\footnotetext{
${ }^{1}$ www.cesga.es
} 
and performances in a computer system, but its flexibility has allowed it to be extended to monitor systems outside the computing realm.

In Fig. 5 a representation of the slow control system is depicted. On the left there are represented the ambient environmental sensors which are read by an Arduino connected via USB to a computer. In this case the temperature probes use a 1-wire bus protocol for communications while the pressure sensor uses the I2C bus. On the right the RPC planes are pictured with their set of embedded sensors: relative humidity, pressure and 9 thermal probes all of them using a I2C bus connected to a Raspberry Pi. The Raspberry also controls, via a serial to USB interface, two high voltage power supplies, one of them providing a positive voltage to the upper plane of the RPC detector and the other a negative voltage to the bottom plane. Both the Rapsberry and the power supplies are encased together. The Raspberry Pi and the server controlling the Arduino are connected to an internal network to a server that provides connectivity to the outside world. The final piece of our system is the Nagios service, installed in an external server it queries the different sensors and power supplies and provides a monitoring and an alert system solution via custom scripts.

\subsection{Calibration and Digitization}

The analysis of the TRAGALDABAS data is done within a specific framework written in C++ and based in ROOT [10]. The events are reconstructed sequentially. First the recorded data is unpacked and converted to physics units following pre-existing tables. Then the time of flight of each hit is fixed within the event. The time resolution after the calibration procedure and other charge corrections is of 340 ps. Then the hits are combined into track candidates which fit the hypothesis that the particle flies at speed of light. Finally all the tracks are combined in order to define an arrival direction of the shower. In case that only one trace is found by reconstruction algorithm that direction is set as the arrival direction of the cosmic. Finally, all the gathered data is collected and prepared for further analysis. At this stage the slow control data, specially the pressure and the temperature in the lab are used to do the correction for obtaining the absolute variation of the rates.

\subsection{Simulation}

In order to to study the interaction of the particles produced by the primary cosmic rays in the atmosphere with the detector and to perform other acceptance studies, among others, a simulation of the detector TRAGALDABAS has been implemented in the ENSARRoot/FAIRRoot framework. ENSARRoot [11] is a derivation of the FAIRRoot framework prepared for the development of small setups and detectors in the Nuclear Physics field in Europe. In FAIRRoot, a ROOT based code, the user can create simulated data and perform analysis within the same framework. Moreover, Geant3 and Geant4 [3] transport engines are supported; however, the user code that creates simulated data does not depend on a particular Monte Carlo engine. The framework delivers base classes which enable the users to construct their detectors and analysis tasks in a simple way. The TRAGALDABAS simulation code derives from the general classes of FAIRRoot, adding and specifying the geometrical, physical description and response of the detectors, event generators for the reactions of interest and analysis and event visualization tools. We are using the simulation also for analysing the impact of the walls, the floors and the roof on the detector. 


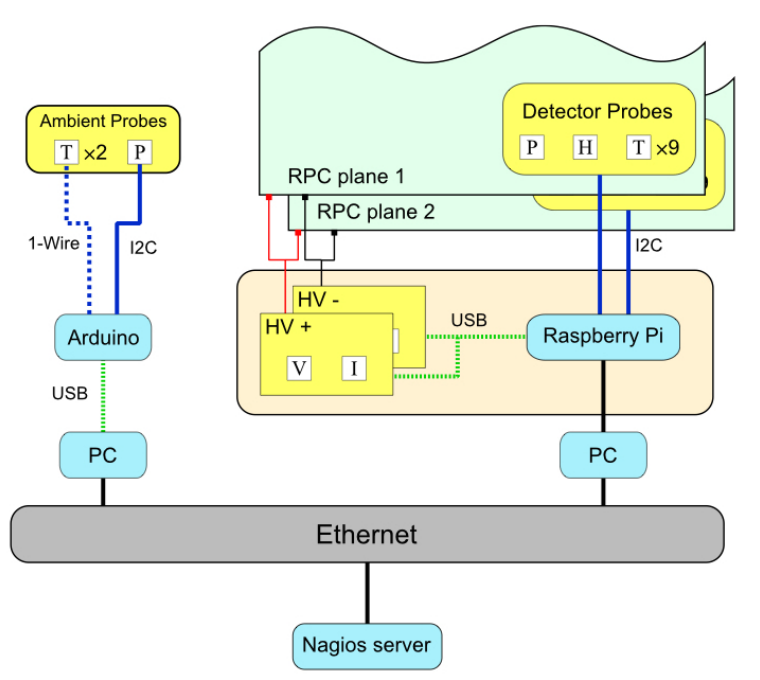

Figure 5: Block diagram of the monitoring of the detector, described in the text.

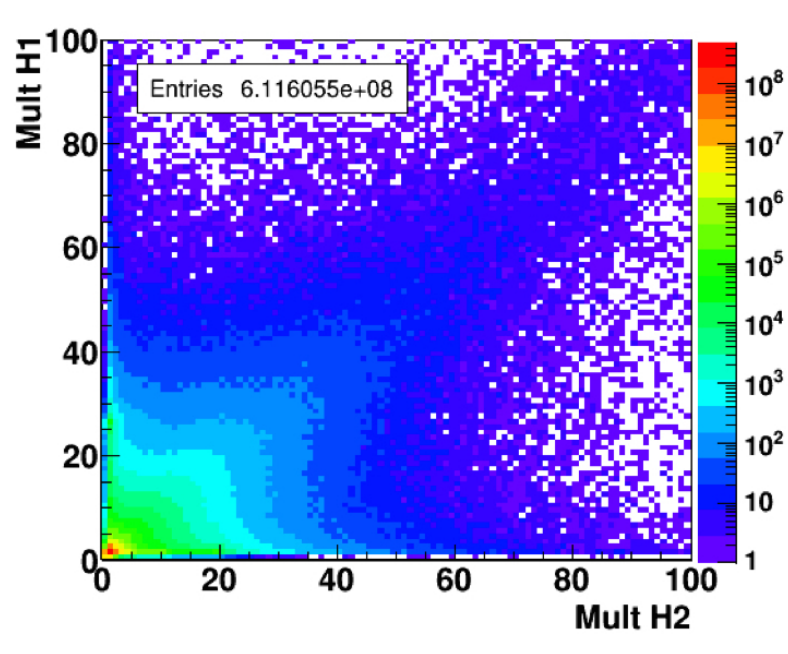

Figure 6: Hit multiplicity of the upper trigger plane versus the one of the lower plane for all the period of data analysed. As can be seen, most of the triggers correspond to one or two particle events. The excess of high multiplicity events near the axis may correspond to streamer events producing a crosstalk domino effect among the cells.

\section{Some preliminary results}

Fig. 6 shows the number of cells fired on the upper plane versus the one of the lower plane used in the trigger for all the period of data analysed. As can be seen, most of the triggers correspond to one or two particle events and the high multiplicity sample is dominated by events with roughly the same number of hits in both planes.

Figure 7 shows some preliminary results obtained from the data taken since the last March. The Forbush Decrease held at June 22nd is apparent in most of the pictures. A very interesting effect observed that should be carefully analized in the future is that, for multiplicities higher than 1 , there is a significant bump of events, starting several days before the decrease.

\section{Summary}

A $1.8 \mathrm{~m}^{2}$ Cosmic Ray detector based on the affordable timing RPC technology, TRAGALDABAS, has been installed at the Univ. of Santiago de Compostela and is being "supported" by a multidisciplinary team in order to understand better the air showers of cosmic origin and to go deeper in the relationship between cosmic rays in a broad range of energies and different phenomena related with the Earth's environment. The preliminary analysis of the rates obtained with different multiplicities and different arrival angles seem to already show very intriguing effects that should be analysed deeper.

\section{Acknowledgments}

We are very grateful to all our colleagues who made possible the development of the detector 
A

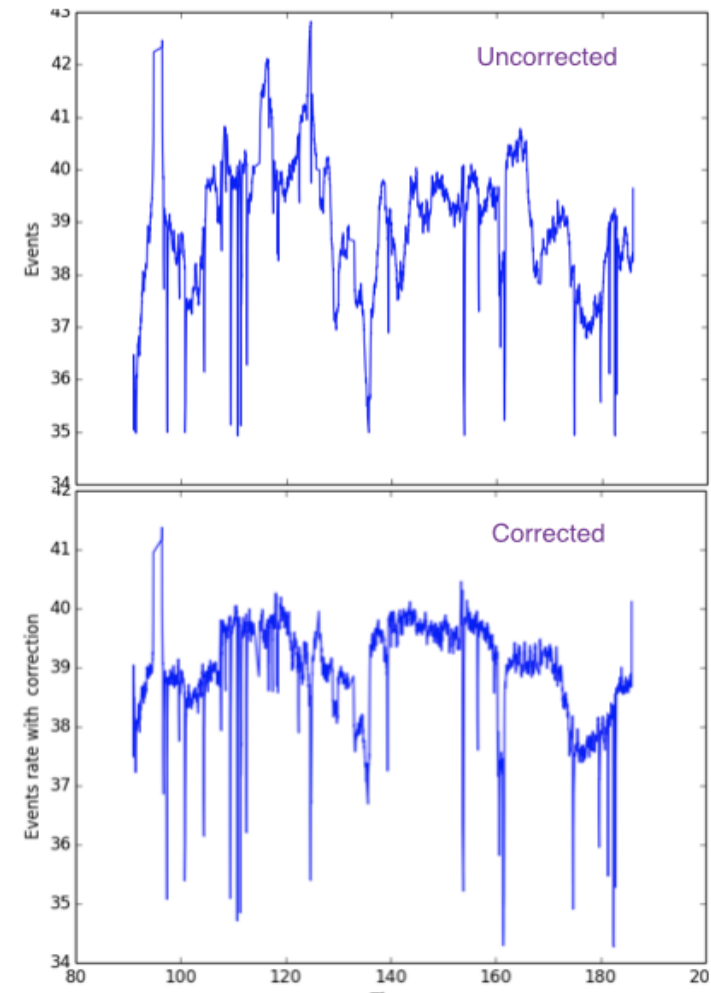

B

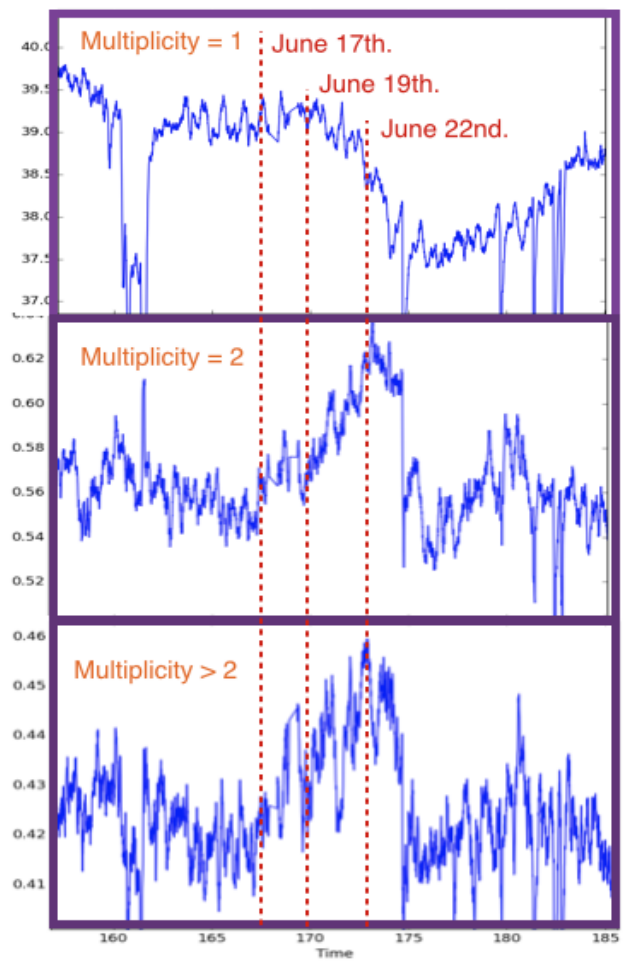

Figure 7: A column shows the rate of measured events with multiplicity $M=1$, both uncorrected and corrected by pressure for the whole data period. Sharp structures correspond either to maintenance or upgrading periods. B columns shows, from top to bottom, the corrected mean rate of events for multiplicities $M=1$, $\mathrm{M}=2$ and $\mathrm{M}>2$, respectively. Vertical dotted lines show the most relevant days.

and its installation in the laboratory of the LabCAF at the Univ. of Santiago de Compostela. In particular we thank M. Traxler, from the GSI-FAIR laboratory for providing us the TRBs we are using in the detector DAQ and the technical staff of LIP-Coimbra and the Graduate students: Juan Fdez. Afonso, Miguel Yermo and Miguel Carballo for their help in the preliminary analysis of the data. We also acknowledge the C. Desarrollo de las Ciencias, of Madrid, for their financial support and all our technical partners: ATI-Coruña, Aplitecs-Biduido and Hydra Technologies-Vigo.

\section{References}

[1] D. Belver et al. Analysis of the space-time microstructure of cosmic ray air showers using the HADES RPC TOF wall, JINST 7 (2012) P10007.

[2] A. Blanco et al. TRAGALDABAS: a new RPC based detector for the regular study of cosmic rays, JINST 9 (2014) C09027.

[3] D. Belver et al. The HADES RPC inner TOF wall, Nucl. Instr. and Meth. A 602 (2012) 687.

[4] D. Belver et al. TRASGO: A proposal for a timing RPCs based detector for analyzing cosmic ray air showers, Nucl. Instrum. \& Meth. A661 (2012) S163-167. 
[5] P. Assis et al. $R \& D$ for an autonomous RPC station in air shower detector arrays, Proc. 32th. International Cosmic Ray Conference V3 (2011) 133-136.

[6] Belver D. et al. Performance of the Low-Jitter High-Gain/Bandwidth Front-End Electronics of the HADES tRPC Wall, IEEE Trans. Nucl. Sci. 57 (2010) 2848-2856.

[7] L. Lópes et al. Study of RPCs for autonomous field stations in cosmic ray research, Proc. of Science, RPC conference 2012043 (2012).

[8] http://www.nagios.org

[9] I. Fröhlich et al. A General Purpose Trigger and Readout Board for HADES and FAIR-Experiments, IEEE Trans. Nucl. Sci. 55 (2008) 59-66.

[10] R. Brun and F. Rademakers ROOT - An object oriented data analysis framework Nuc. Inst. Meth. A 389 (1997) 81Đ86. See also: http://root.cern.ch/

[11] http://igfae.usc.es/sinurse/index.php/downloadmenuitem

[12] S. Agostinelli et al. Geant4 - a simulation toolkit Nuc. Inst. Meth. A 506 (2003) 250.

\section{The TRAGALDABAS Collaboration:}

Members: H. Alvarez Pol(9), A. Blanco (4), J.J. Blanco (1), J. Collazo (8), J. Díaz Cortés (8), P. Fonte (4), D. García Castro(8), J.A. Garzón (8), A. Gómez-Tato (7), M. González (8), G. Kornakov(6), T. Kurtukian (2), R. Lorenzo (9), J.M. López (8), L. Lópes (4), M. Morales (8), A.Morozova (3), J.C. Mouriño (7), M.A. Pais (3), M. Palka (5), V. Pérez Muñuzuri (10), P. Rey(7), P. Ribeiro (3), M. Seco (9), J. Taboada (10).

Institutions: 1. Univ. Alcalá /Alcalá de Henares, Spain. 2. CEN-Bordeaux, France. 3. CGUCUniv. de Coimbra / Coimbra, Portugal. 4. LIP-Coimbra / Coimbra, Portugal. 5. Jagellionan Univ. /Cracow, Poland. 6. TU-Darmstadt, Germany. 7. CESGA/ S. de Compostela, Spain. 8. LabCAFUniv. S. de Compostela / S. de Compostela, Spain. 9. Particle Phys. Dept. Univ. S. de Compostela / S. de Compostela, Spain. 10. Meteogalicia-Xunta de Galicia / S. de Compostela, Spain. 